\title{
High-latitude electromagnetic and particle energy flux during an event with sustained strongly northward IMF
}

\author{
H. Korth ${ }^{1}$, B. J. Anderson ${ }^{1}$, H. U. Frey ${ }^{2}$, and C. L. Waters ${ }^{3}$ \\ ${ }^{1}$ The Johns Hopkins University, Applied Physics Laboratory, Laurel, Maryland, USA \\ ${ }^{2}$ Space Sciences Laboratory, University of California Berkeley, California, USA \\ ${ }^{3}$ School of Mathematical and Physical Sciences, The University of Newcastle, NSW, Australia
}

Received: 13 October 2004 - Revised: 21 January 2005 - Accepted: 8 February 2005 - Published: 3 June 2005

\begin{abstract}
We present a case study of a prolonged interval of strongly northward orientation of the interplanetary magnetic field on 16 July 2000, 16:00-19:00 UT to characterize the energy exchange between the magnetosphere and ionosphere for conditions associated with minimum solar windmagnetosphere coupling. With reconnection occurring tailward of the cusp under northward IMF conditions, the reconnection dynamo should be separated from the viscous dynamo, presumably driven by the Kelvin-Helmholtz (KH) instability. Thus, these conditions are also ideal for evaluating the contribution of a viscous interaction to the coupling process. We derive the two-dimensional distribution of the Poynting vector radial component in the northern sunlit polar ionosphere from magnetic field observations by the constellation of Iridium satellites together with drift meter and magnetometer observations from the Defense Meteorological Satellite Program (DMSP) F13 and F15 satellites. The electromagnetic energy flux is then compared with the particle energy flux obtained from auroral images taken by the far-ultraviolet (FUV) instrument on the Imager for Magnetopause to Aurora Global Exploration (IMAGE) spacecraft. The electromagnetic energy input to the ionosphere of $51 \mathrm{GW}$ calculated from the Iridium/DMSP observations is eight times larger than the $6 \mathrm{GW}$ due to particle precipitation all poleward of $78^{\circ}$ MLAT. This result indicates that the energy transport is significant, particularly as it is concentrated in a small region near the magnetic pole, even under conditions traditionally considered to be quiet and is dominated by the electromagnetic flux. We estimate the contributions of the high and mid-latitude dynamos to both the Birkeland currents and electric potentials finding that highlatitude reconnection accounts for $0.8 \mathrm{MA}$ and $45 \mathrm{kV}$ while we attribute $<0.2 \mathrm{MA}$ and $\sim 5 \mathrm{kV}$ to an interaction at lower latitudes having the sense of a viscous interaction. Given that these conditions are ideal for the occurrence of the $\mathrm{KH}$ instability at the magnetopause and hence the viscous interaction,
\end{abstract}

Correspondence to: H. Korth

(haje.korth@jhuapl.edu) this result suggests that the viscous interaction is a small contributor to coupling solar wind energy to the magnetosphereionosphere system.

Keywords. Ionosphere (Polar ionosphere; Electric fields and currents; Ionosphere-magnetosphere interactions)

\section{Introduction}

Solar wind-magnetosphere coupling during intervals when the interplanetary magnetic field (IMF) is northward is fundamentally different from that during southward IMF, and such conditions offer unique opportunities to characterize the coupling process. Following the paradigm of anti-parallel merging (Crooker, 1979), reconnection occurs at latitudes poleward of the cusp during northward IMF giving rise to a pair of high-latitude convection cells with reversed sense of rotation, i.e. the plasma flow is sunward at the poleward boundary of the convection cells (Russell, 1972; Maezawa, 1976; Reiff and Burch, 1985). Convection of charged particles in a magnetic field is associated with the formation of electric fields and currents, which intimately couple the ionosphere to the magnetosphere. How much energy is transferred to the ionosphere under these conditions is not well known since the currents and electric fields occur poleward of the vast majority of auroral observatories. Moreover, time variations in the interplanetary magnetic field lead to reconfigurations of the convection so that it can be difficult to uniquely identify the conditions associated with nearly pure northward IMF.

The major mechanisms of energy transfer at the ionosphere are particle precipitation and exchange of electromagnetic energy flux. Precipitating energetic particles induce ionization and heating via collisions, and the energy deposited in this process can be quantified by remote-sensing far-ultraviolet emissions (Rees et al., 1988; Lummerzheim et al., 1997). On the other hand, electromagnetic energy transferred from the magnetosphere to the ionosphere is 
dissipated as Joule heating (e.g., Cole, 1962) or converted into mechanical energy of the neutral gas (e.g., Thayer et al., 1995; Fujii et al., 1999). Evaluating the relative contributions of particle and electromagnetic energy transport to the ionosphere is central to understanding the effects of the energy on the dynamics of the ionosphere and thermosphere. The energy flux during northward IMF is typically thought to be low, but there are very few observations providing coverage of the region near the magnetic pole where the energy deposition probably occurs.

Determining the distribution of electromagnetic energy flux is not trivial and typically requires coordinated observations over broad regions. The radial Poynting vector $S_{\mathrm{r}}$ can be determined directly from measurements of horizontal electric fields $\boldsymbol{E}$ and magnetic perturbations $\boldsymbol{b}=\boldsymbol{B}-\boldsymbol{B}_{0}$ :

$\boldsymbol{S}_{\mathrm{r}}=\frac{1}{\mu_{0}} \boldsymbol{E} \times \boldsymbol{b}$,

where the magnetic perturbations are deviations of the observed magnetic field $\boldsymbol{B}$ from a main field model $\boldsymbol{B}_{0}$ and are produced by the currents in the system. Since the magnetic perturbations required in Eq. (1) are those measured above the ionosphere, direct evaluation of $S_{\mathrm{r}}$ requires observations from satellites. Both event studies (e.g., Sugiura, 1984; Erlandson et al., 1990; Gary et al., 1994; Vaivads et al., 2003) and statistical studies (e.g., Gary et al., 1994; Keiling et al., 2003) have previously been performed, but none of these studies isolated strongly northward IMF conditions. Satellite event studies typically focus on the Poynting vector along a single satellite trajectory and are unable to provide the global picture of the ionospheric energy deposition. On the other hand, global distributions presented in statistical studies are only valid in an average sense and almost certainly differ significantly from those observed during individual events. Recently, Waters et al. (2004) used magnetometer data from the Iridium constellation together with electric field results from the Super Dual Auroral Radar Network (SuperDARN) to obtain global distributions of electromagnetic energy flux for two events occurring for southward IMF. Their findings indicate enhanced flux in the auroral zones together with a broad region of flux over the polar cap. They obtained total energy fluxes of 40 to $50 \mathrm{GW}$ for two moderately active periods.

Alternate estimates of the global electromagnetic energy input have been obtained from ground-based magnetometer (Förster et al., 1991) and radar (Thayer, 1998a) networks or a combination thereof (Lu et al., 1995) using Poynting's theorem, under time-stationary conditions given by

$\nabla \cdot S=-\boldsymbol{J} \cdot \boldsymbol{E}$.

In Eq. (2), $\boldsymbol{J} \cdot \boldsymbol{E}$ is the electromagnetic energy transfer rate, which is further converted into both Joule heating rate and mechanical energy transfer rate as stated above. Typical energy deposition rates derived from this method are $50 \mathrm{GW}$ during moderate activity to several hundred GW during high geomagnetic activity (Lu et al., 1995; Thayer, 1998b; Fujii et al., 1999). The ability to establish the partitioning between the two components is a particular strength of the radar technique (Fujii et al., 1999). However, all methods using ground observations to infer the electromagnetic energy flux suffer from the paucity of observations in regions of the magnetic poles. The region poleward of $80^{\circ}$ latitude is where the Birkeland currents occur for strongly northward IMF (Iijima et al., 1984; Zanetti et al., 1984; Iijima and Shibaji, 1987; Zanetti et al., 1990), so under these conditions we expect the bulk of the energy transfer between the ionosphere and the magnetosphere to occur here.

Study of the energy transport for strongly northward IMF conditions is also particularly interesting because it provides a unique opportunity to determine the relative contributions of reconnection and the viscous interaction to solar windmagnetosphere coupling. Two main processes have been proposed for the transport of energy and momentum from the solar wind into the magnetosphere: Reconnection of the interplanetary magnetic field lines with the Earth's magnetic field (Dungey, 1961) and viscous interaction between the solar wind flow and the magnetospheric circulation (Axford and Hines, 1961). Since the introduction of these mechanisms, there has been debate over the significance of the contribution of viscous interaction to magnetospheric dynamo processes (Rostoker et al., 1987; Heikkila, 1990). For example, Reiff et al. (1981) report a significant $(35 \mathrm{kV})$ persistent potential drop due to viscous interaction, while Mozer (1984) attributes at most $10 \%$ (5 to $15 \mathrm{kV}$ ) of the magnetospheric electric potential to viscous interaction. Wygant et al. (1983) demonstrate that the contributions to the polar cap potential not associated with the reconnection process can be limited to less than $20 \mathrm{kV}$. Northward IMF conditions should be favorable for unambiguously identifying the viscous dynamo contribution for two reasons. First, the viscous interaction is believed to be primarily driven by the Kelvin-Helmholtz $(\mathrm{KH})$ instability, and the conditions for instability are most favorable when the IMF is oriented northward or southward (Kivelson and Chen, 1995). Furthermore, the reconnection and viscous dynamos are spatially separated under northward IMF conditions, allowing one to assess their relative contributions.

We present here the study of a time period of prolonged northward IMF on 16 July 2000, to assess the contributions of particle precipitation and electromagnetic energy flux to the total ionospheric energy input and to estimate the contribution of the viscous interaction. We determine the radial Poynting vector by combining the global observations of the magnetic perturbations by the constellation of Iridium satellites, DMSP magnetic field and plasma drift observations, and a statistical model for the ionospheric conductivity. The data sets are described in Sect. 2 together with an overview of the Birkeland currents and auroral emissions that occurred during the event. Section 3 describes the technique used to evaluate the distribution of the Poynting vector radial component and presents our results for the electromagnetic energy flux. Section 4 addresses the analysis of the auroral emissions to determine the precipitating particle energy flux and compares it with the electromagnetic energy flux. The evidence for the presence of a solar wind-magnetosphere 
viscous interaction is considered in Sect. 5 and the results are summarized in Sect. 6 .

\section{Data sets and event overview}

\subsection{Iridium data}

The engineering magnetometer data from the Iridium satellites is one of the key data sets used for this analysis. The Iridium network consists of 79 spacecraft distributed over six orbit planes in 780-km altitude circular near polar orbits. Each satellite is equipped with a 30-nT resolution engineering magnetometer, which can be used to detect magnetic perturbations due to Birkeland currents. These data are routinely used to derive global maps of Birkeland currents (Anderson et al., 2000; Waters et al., 2001). An intermediate product in the derivation of the currents is the distribution of the horizontal vector magnetic perturbations, $\boldsymbol{b}$, due to the Birkeland currents and is associated with the poloidal ionospheric current (Waters et al., 2001). The $\boldsymbol{b}$-distribution is used here in the estimation of the Poynting vector. Due to the coarse time sampling of the Iridium engineering magnetometer data, on average about $200 \mathrm{~s}$ between samples on an individual satellite, roughly one hour of data is required to obtain a latitude resolution of approximately $4^{\circ}$ in the Iridium fit results (Anderson et al., 2000; Korth et al., 2004; Waters et al., 2004).

\subsection{Event selection}

Because we must accumulate Iridium data over periods of an hour or longer, we need to select events for which the solar wind driving is quasi time stationary. To do this, the solar wind and IMF conditions are analyzed to select candidate time periods. We use the Level 2 data of the magnetometer (MAG) and Solar Wind Electron Proton Alpha Monitor (SWEPAM) (Smith et al., 1998; McComas et al., 1998) aboard the Advanced Composition Explorer (ACE) spacecraft located at the first Lagrangian point, L1. The following criteria are used in searching the ACE magnetometer data to identify periods of stable IMF: 1) The deviation of the IMF orientation from the average direction must be less than $\pm 25^{\circ}$ for at least three hours; 2) The average IMF magnitude must be at least $5 \mathrm{nT}$ to ensure that the Birkeland currents are relatively strong; and 3) The IMF cone angle must be between $45^{\circ}$ and $135^{\circ}$ ensuring quasi-perpendicular bow shock conditions to minimize time variations in magnetospheric forcing due to shock dynamics. Because the resulting intervals are at least three hours long, the errors introduced by assuming simple advection from the L1 point to Earth are negligible relative to the period available for study.

An interval of northward IMF which satisfies all of the above criteria and yields reliable results from Iridium was observed by ACE on 16 July 2000, from 15:32 UT to 18:50 UT, during the early recovery from the geomagnetic superstorm associated with the so-called Bastille day solar flare and coronal mass ejection (CME) (Aulanier et al., 2000; Jordanova et al., 2001). The solar wind and IMF conditions at

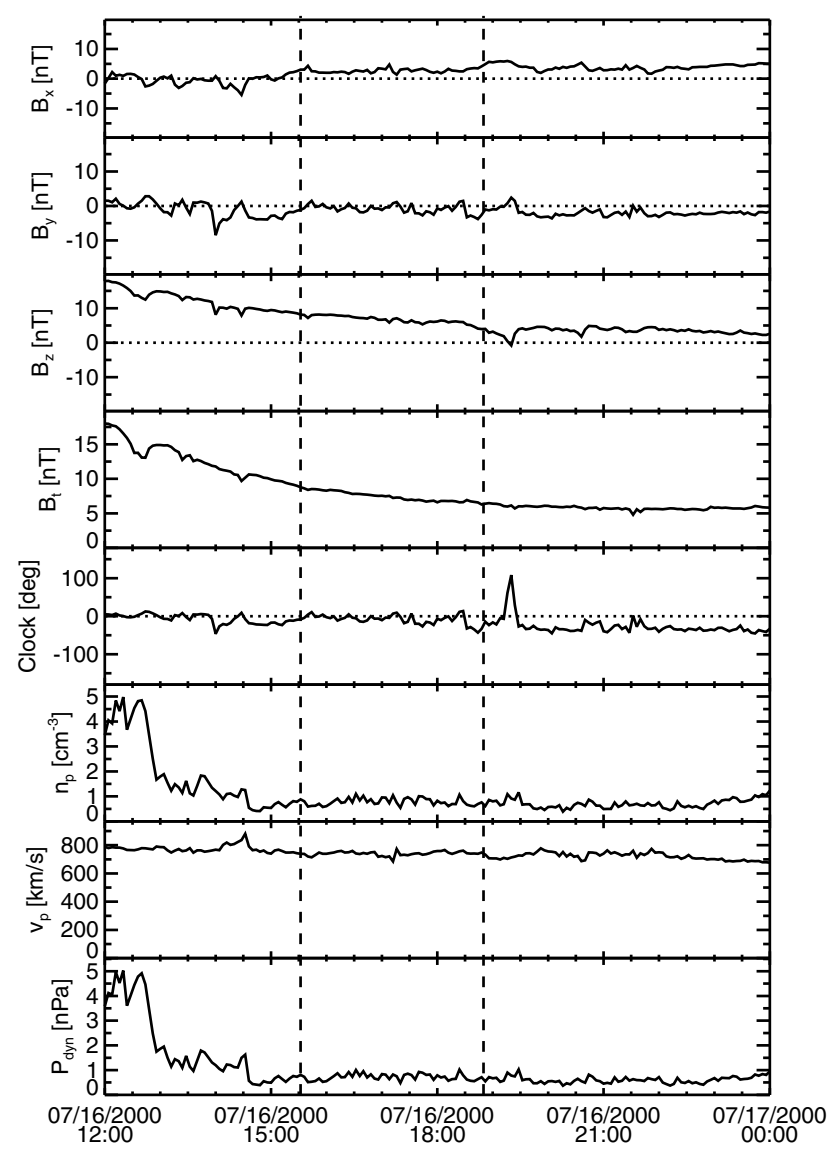

Fig. 1. Solar wind and IMF conditions on 16 July 2000, as observed by ACE in GSM coordinates. The interval of stable IMF conditions is marked by vertical dashed lines. See text for details.

ACE are shown in Fig. 1, where the event interval is marked by two vertical dashed lines. From top to bottom the panels show the IMF $B_{\mathrm{x}}, B_{\mathrm{y}}$ and $B_{\mathrm{z}}$ components in GSM coordinates; the IMF magnitude, $B_{\mathrm{t}}$; the IMF clock angle defined by $\arctan \left(B_{\mathrm{y}} / B_{\mathrm{z}}\right)$; the proton number density, $n_{\mathrm{p}}$; the proton bulk speed, $v_{\mathrm{p}}$; and the proton dynamic pressure, $p_{\text {dyn }}$. The time averages and standard deviations for the IMF magnitude and its three GSM components are $B_{\mathrm{t}}=7.4 \pm 0.7 \mathrm{nT}$, $B_{\mathrm{x}}=2.7 \pm 0.7 \mathrm{nT}, \quad B_{\mathrm{y}}=-0.9 \pm 1.3 \mathrm{nT}$, and $B_{\mathrm{z}}=6.7 \pm 1.1 \mathrm{nT}$. This corresponds to an IMF clock angle of $-8.4^{\circ}$ with respect to the northward direction. The average solar wind proton density is $0.8 \mathrm{~cm}^{-3}$ and the mean solar wind flow speed is $740 \mathrm{~km} / \mathrm{s}$, giving a time lag for the arrival of these conditions at Earth of $34 \mathrm{~min}$. This establishes the period of interest at Earth from 16:06 UT to 19:24 UT.

\subsection{DMSP and IMAGE/FUV data}

Estimating energy flux requires information in addition to the magnetic perturbations from Iridium. In prior analyses we have combined results from Iridium and SuperDARN to provide distributed observational inputs for both $\boldsymbol{b}$ and $\boldsymbol{E}$ in Eq. (1) (Waters et al., 2004). For this event however the SuperDARN returns in the region of interest were too limited 


\section{7/16/2000 17:00-18:00 UT (North)}
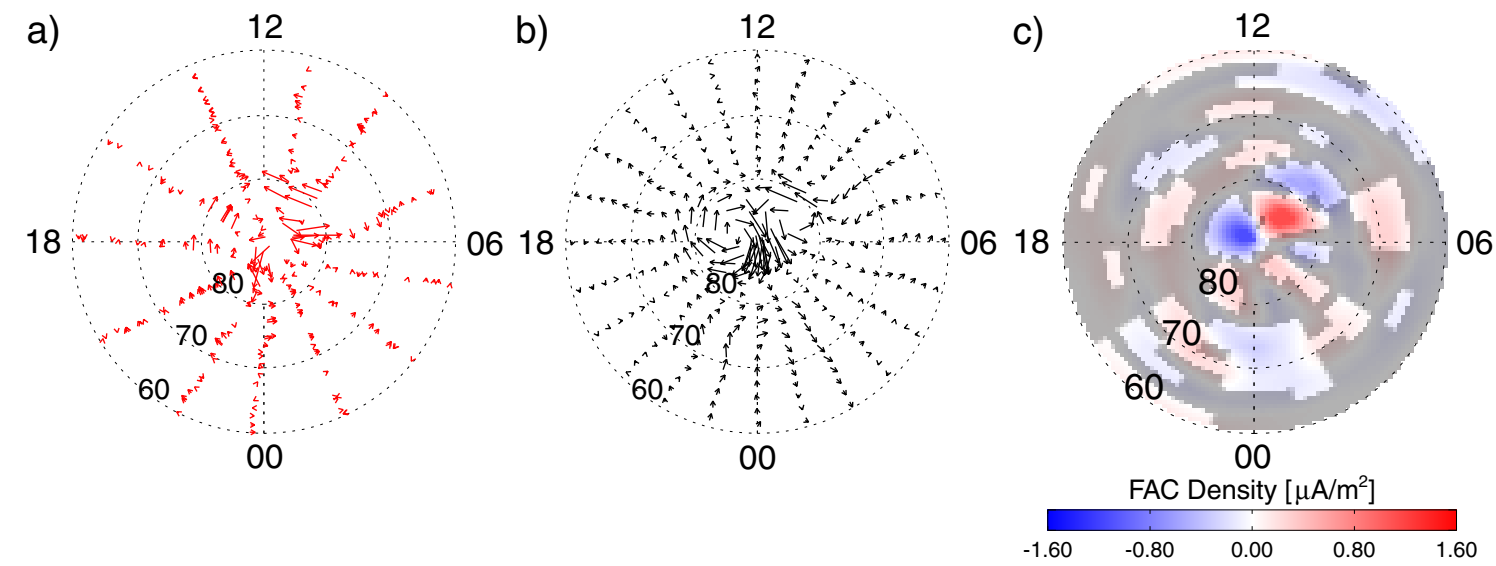

Fig. 2. Iridium observations and derived quantities for 16 July 2000, from 17:00-18:00 UT in AACGM MLT-MLAT coordinates: (a) Iridium samples of the magnetic field perturbations, (b) their spherical harmonic fit, and (c) the derived distribution of field-aligned currents. Upward (downward) Birkeland current are shown in red (blue). Birkeland currents below the quiet-time baseline are masked in gray.

to use this technique, so we took a different approach applicable to this event. Because the event occurred during northern summer and at times of large dipole tilt, 16:00 UT to 19:00 UT, the midnight terminator at ionospheric altitudes was near $60^{\circ}$ MLAT, and the ionospheric conductivity in the region of interest, poleward of $70^{\circ}$ MLAT, is therefore primarily due to EUV illumination. For this case then, we can use the Iridium magnetic perturbations and a model EUV conductance to determine the spatial distribution of the steady state convection fields.

Magnetometer and plasma drift data were available from the DMSP F13 and F15 satellites, and these data are used both to confirm the patterns and to quantitatively correct (rescale) the $\boldsymbol{b}$ and $\boldsymbol{E}$ estimated from the Iridium data and the conductance model. The DMSP satellites in 840-km altitude sun-synchronous circular orbits provide sampling on tracks approximately along the 18:00-06:00 MLT meridian (F13) and 21:00-09:00 MLT meridian (F15). The sunlit conditions for this event are optimal for DMSP plasma drift measurements because the ionospheric densities are relatively high. The plasma drift data quality in the Northern Hemisphere is excellent for this event. The DMSP magnetometer data is used to re-scale the $\boldsymbol{b}$-distribution derived from Iridium to correct the fit results, which we expect underestimate the true perturbations (Sect. 3.2). The plasma drift data is used to derive a correction for the conductance model (Sect. 3.3).

To compare the electromagnetic energy flux with the energy flux of the precipitating particles we use observations by the Wideband Imaging Camera (WIC) (Mende et al., 2000a) of the Far-Ultraviolet (FUV) instrument (Mende et al., 2000b) aboard the IMAGE satellite. This instrument also provides information regarding proton precipitation in the SI12 spectrograph, which is used to confirm whether the auroral emissions are primarily caused by electrons or also have a significant ion component. Finally, DMSP SSJ/4 particle data (Hardy et al., 1984) are used to provide additional confirmation of the precipitating particles and to estimate their characteristic energy. This characteristic energy is required to convert the auroral luminosity to particle energy flux (Frey et al., 2003).

\subsection{Field-aligned currents from iridium observations}

The Northern Hemisphere Iridium observations for the one hour period from 17:00 UT to 18:00 UT during the event are shown in Fig. 2. All satellite data are presented in AACGM coordinates (Baker and Wing, 1989). Figure 2 shows MLTMLAT distributions of (a) the original input cross-track magnetic field perturbations, (b) the spherical harmonic fit, $\boldsymbol{b}$, and (c) the Birkeland currents evaluated applying Amperes law to the $\boldsymbol{b}$-distribution. The vector fit perturbations, $\boldsymbol{b}$, are mapped from the Iridium satellites altitude $(780 \mathrm{~km})$ to ionospheric altitudes $(120 \mathrm{~km})$ using a $r^{-3 / 2}$ scaling to account for the convergence of the flux tubes with decreasing altitude. The same scaling formula is applied below to magnetometer data from the DMSP satellites ( $840 \mathrm{~km}$ altitude). The most prominent feature in Fig. $2 b$ is a double vortex in the magnetic field signature in the dayside ionosphere near the geomagnetic pole, which corresponds to the pair of strong, oppositelydirected Birkeland currents seen in Fig. 2c. These currents are commonly observed during intervals of strongly northward IMF and have previously been termed NBZ currents (Iijima et al., 1984; Zanetti et al., 1984; Iijima and Shibaji, 1987). The NBZ currents are directed upward (red) on the dawn side and downward (blue) in the dusk side. Regions where the current densities are lower than two standard deviations above the quiet-time noise level are shaded gray.

The noise level was established from thirty independent intervals with prolonged periods of very low activity. For each quiet-time event, the mean of the average upward and downward current density was evaluated in $5^{\circ}$-wide annuli in magnetic latitude. The quiet-time noise is the average of the 
current density magnitudes over the events. The standard deviation in each latitude bin is used to estimate confidence levels relative to the noise level. Figure 3 shows the noise level versus co-latitude together with its standard deviation (error bars) and the average positive and negative current densities for the 17:00-18:00 UT interval on 16 July 2000. The noise level is largest near the pole, $0.16 \mu \mathrm{A} / \mathrm{m}^{2}$, and decreases toward the equator, reaching $<0.05 \mu \mathrm{A} / \mathrm{m}^{2}$ near $40^{\circ}$ MLAT. The latitude dependence arises because the tracks are closer together at higher latitudes, which translates into larger noise in the azimuthal gradients and hence in the derived current densities (cf. Korth et al., 2004). The noise level may be somewhat overestimated at high latitudes because minor contributions from true NBZ currents may have been included in the analysis. The dotted line shows a second order polynomial fit to the noise level. We use two standard deviations above the noise level to indicate Birkeland currents that are statistically significant. The Birkeland currents poleward of $\sim 75^{\circ}$ are considerably stronger than the noise level, but below that the currents are either completely consistent with quiet noise levels or only marginally larger.

\subsection{Auroral emissions}

Auroral emissions provide a way to assess both the time stationarity of the magnetosphere-ionosphere interaction as well as the particle precipitation input. In this section we present the auroral emissions as observed by IMAGE FUV/WIC demonstrating the stability of the system over this time and the relationship of the emissions to the Birkeland currents. The emissions will be used later to estimate the total precipitating energy flux for comparison with the total electromagnetic energy flux. Figure 4 shows snapshots of the aurora taken by IMAGE FUV/WIC between 16:00 UT and 19:00 UT on 16 July, 2000. The WIC instrument is sensitive to wavelengths between $140 \mathrm{~nm}$ and $190 \mathrm{~nm}$. The FUV data are corrected for instrument background, $\sim 500$ counts, and dayglow emissions, $\sim 4000$ counts. The auroral images show a localized region of intense emissions near the pole in the pre-noon sector that persists for the entire 3-h interval. This is consistent with the stability of the system indicated by the IMF and Iridium data, and also demonstrates that the system exhibited only modest departures from the average configuration over the time period with no other major auroral features occurring on shorter time scales. To compare the auroral emissions with the Birkeland currents, we construct one-hour averages of the WIC images from $\sim 30$ snapshots taken approximately two minutes apart, each of which is registered in magnetic latitude and local time. Figure 5 shows the Birkeland currents (upper panels) and one-hour averaged WIC auroral emissions (bottom panels) from 16:00 UT to 19:00 UT. In all three hours the NBZ currents dominate the Birkeland currents, and the single prominent feature in the averaged auroral emissions is the pre-noon emission at about $85^{\circ}$ MLAT. In every case the auroral emissions are coincident with the upward NBZ current derived from Iridium. One expects upward currents carried by electrons to be associated
Average Current Density: 16 July 2000 1700-1800

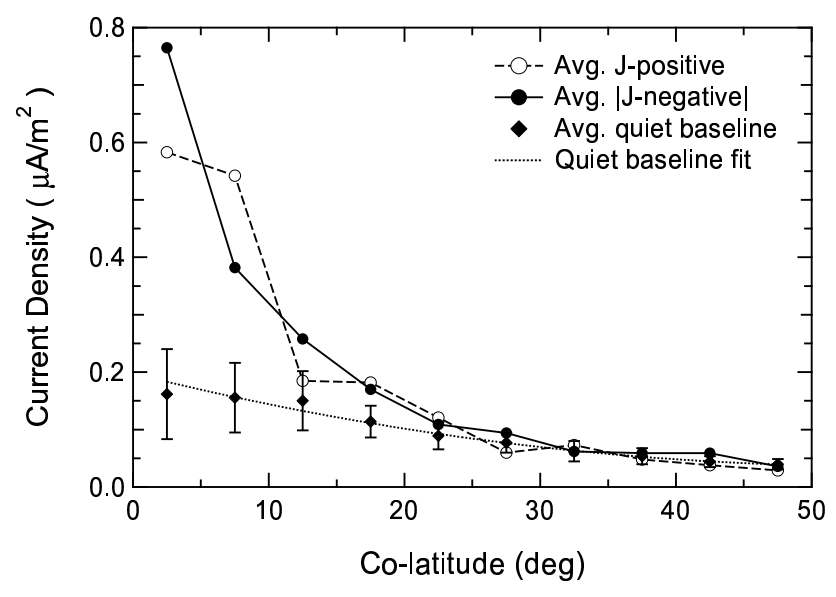

Fig. 3. Local time averaged distribution of the upward (open circles) and downward (closed circles) Birkeland currents on 16 July 2000, 16:00-17:00 UT. The quiet-time noise level is represented by diamonds.

with auroral emissions, so this result provides confirmation of the Iridium derived current pattern.

\section{Electromagnetic energy flux estimation}

Estimating the Poynting vector requires knowledge of the electric field as well as the magnetic perturbations. In other cases we have used SuperDARN to estimate the electric field (Waters et al., 2004), but as stated above in this event the radar returns are too sparse for this analysis. Instead, we take here advantage of excellent data from the DMSP drift meter and magnetometer instruments, favorable DMSP tracks through the regions of Birkeland currents and auroral emissions, and the sunlit ionospheric conditions (northern summer) that favor a solar EUV dominated ionospheric conductivity. Because the ionosphere is sunlit, we expect that the conductance will be well represented by a statistical model. The analysis proceeds as follows. We first compare the DMSP magnetic field data with the Iridium $\boldsymbol{b}$ results. This gives us a correction factor, $c_{\mathrm{b}}$, defined and evaluated in Sect. 3.2, which we apply to the $\boldsymbol{b}$ values. We then use the conductance model to derive an electric field from the Iridium $\boldsymbol{b}$ pattern. The cross track drift implied by this electric field is compared with DMSP observations to provide a correction to the conductance, $c_{\Sigma}$, defined and evaluated in Sect. 3.3. We use the Iridium $\boldsymbol{b}$ pattern and conductance model together with both correction factors, $c_{\mathrm{b}}$ and $c_{\Sigma}$, to obtain the best possible estimate for the distribution of radial Poynting vector for this event. Finally, we evaluate the total electromagnetic energy flux.

\subsection{Electric field}

A reliable estimate of the ionospheric conductivity allows one to use the magnetic perturbations to derive the electric 


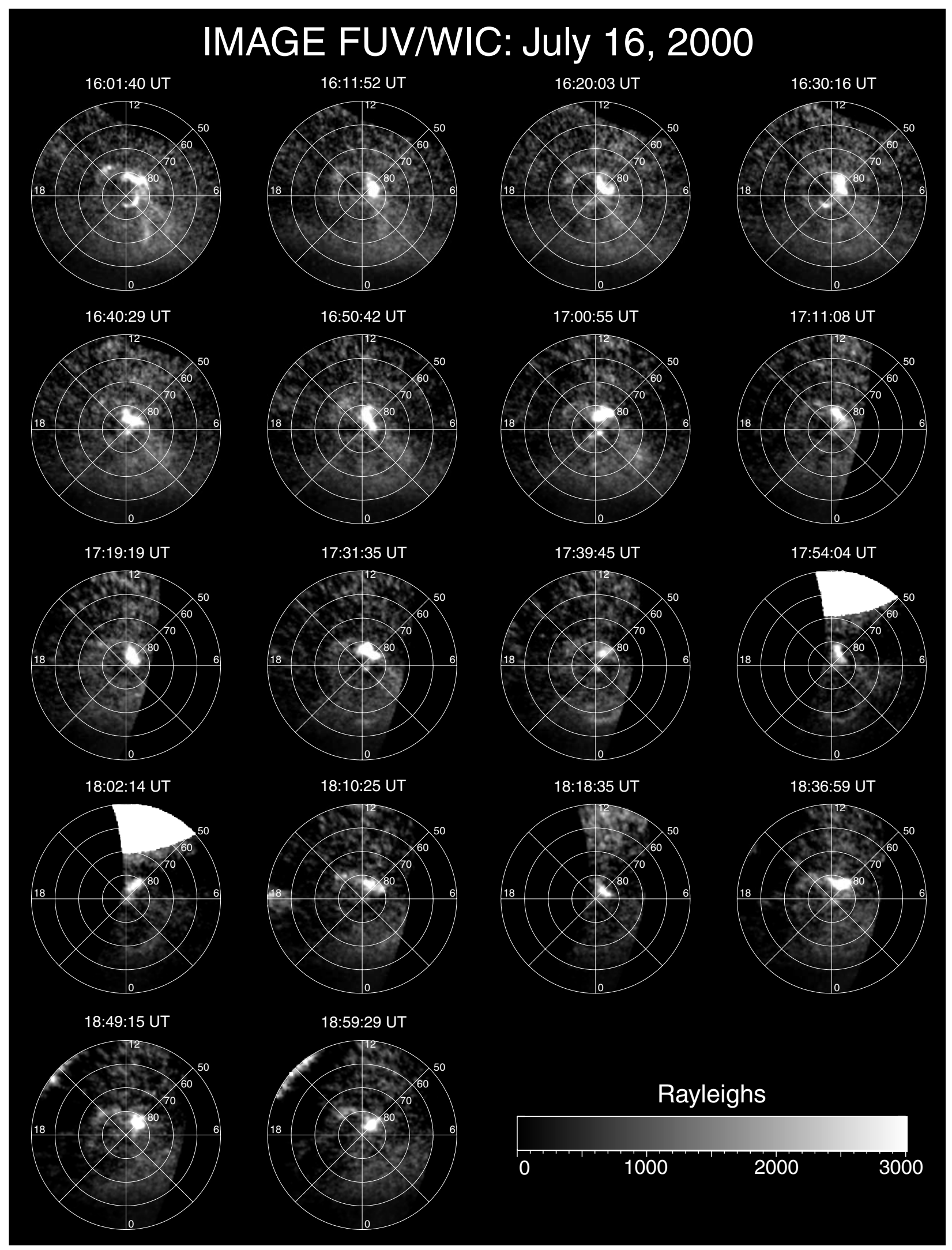

Fig. 4. Auroral emissions in AACGM MLT-MLAT coordinates observed by IMAGE FUV/WIC on 16 July 2000, between 16:00 UT and 19:00 UT. 


\section{7/16/2000 (North)}
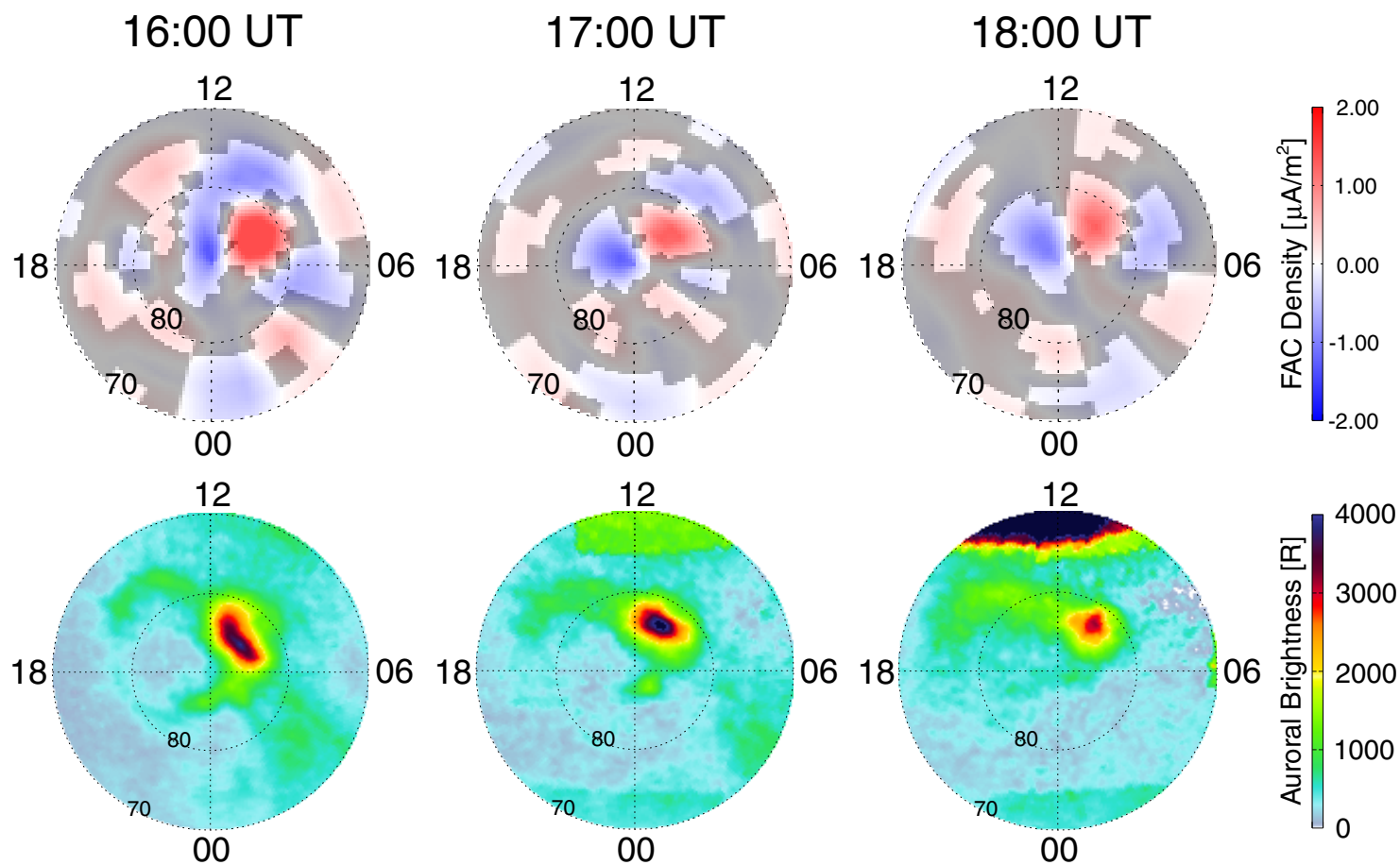

Fig. 5. Birkeland currents (upper panels) and one-hour averaged WIC auroral emissions (bottom panels) on 16 July 2000, from 16:00 UT to 19:00 UT in AACGM MLT-MLAT coordinates.

field. If one assumes that the conductivity gradients are small, that the system is stationary in time, and that the neutral wind effects are small, then given the height-integrated Pedersen conductivity $\Sigma_{\mathrm{P}}$, the electric field vector $\boldsymbol{E}$ can be expressed from the fitted magnetic field data $\boldsymbol{b}$ as

$\boldsymbol{E}=\frac{1}{\mu_{0} \Sigma_{\mathrm{P}}} \hat{\boldsymbol{r}} \times \boldsymbol{b}$

where $\hat{\boldsymbol{r}}$ is the radial unit vector. Equation (3) is obtained by applying Ampere's law to the horizontal ionospheric current. In dividing by $\Sigma_{\mathrm{P}}$ to obtain $\boldsymbol{E}$ the tilt of the magnetic field is ignored, an approximation that is accurate to better than $3 \%$ poleward of $60^{\circ}$ MLAT, where the average deviation between the true magnetic field vector and the radial direction is $<10^{\circ}$ in a dipolar field (Ratcliffe, 1972). The sources for ionospheric conductivity are solar illumination, particle precipitation, and background radiation (e.g., Reiff, 1983). For 16:00 UT to 19:00 UT on 16 July 2000, the terminator at ionospheric altitudes at midnight is between $60^{\circ}$ and $62^{\circ}$ MLAT, so solar EUV should be the dominant source of ionization in the region of interest. We use the statistical model of Rasmussen et al. (1988), where $\Sigma_{\mathrm{P}}$ in units of S is given by

$\Sigma_{\mathrm{P}}=\frac{4.5}{B}\left(1-0.85 v^{2}\right)\left(1+0.15 u+0.05 u^{2}\right)$,

where $B$ is the magnetic field strength in Gauss, $\nu=\chi / 90^{\circ}$ depends on the solar zenith angle $\chi$ in degrees, and
$u=F_{10.7} / 90$ is a function of the $10.7-\mathrm{cm}$ solar radio flux in units of $10^{-22} \mathrm{~W} \mathrm{~m}^{-2} \mathrm{~Hz}^{-1}$. This model is valid sunward of the terminator at ionospheric altitudes. Along the noonmidnight meridian at $70^{\circ}$ MLAT on the dayside, the magnetic pole, and $70^{\circ}$ MLAT on the nightside (sunward of the terminator), the Rasmussen et al. model yields $\Sigma_{\mathrm{P}}=10.1 \mathrm{~S}$, 7.1 S, and 2.4 S, respectively. Since the NBZ currents are on the dayside, the EUV contribution to the conductivity in the region of interest is quite large, giving a minimum $\Sigma_{P}$ of about $7 \mathrm{~S}$. The radial Poynting vector is obtained by inserting Eq. (3) into Eq. (1):

$S_{\mathrm{r}}=-\frac{b^{2}}{\mu_{0}^{2} \Sigma_{\mathrm{P}}}$.

$S_{\mathrm{r}}$ is purely downward, a result that follows from taking the electric field to be proportional to the current and hence assuming that the ionosphere is dissipative.

\subsection{DMSP comparison: Magnetic fields}

To verify that this simplified approach yields reliable results, we compare the Iridium $\boldsymbol{b}$ and the drift implied by Eq. (3) to those observed from the DMSP F13 and F15 satellites. The Iridium $\boldsymbol{b}$ fits are known to underestimate the true peak magnetic perturbations. Two factors contribute to this. First, the spherical harmonic fit cannot resolve features shorter than the shortest wavelength basis function. Second, the coarse sampling in time and hence in latitude along the orbit track 


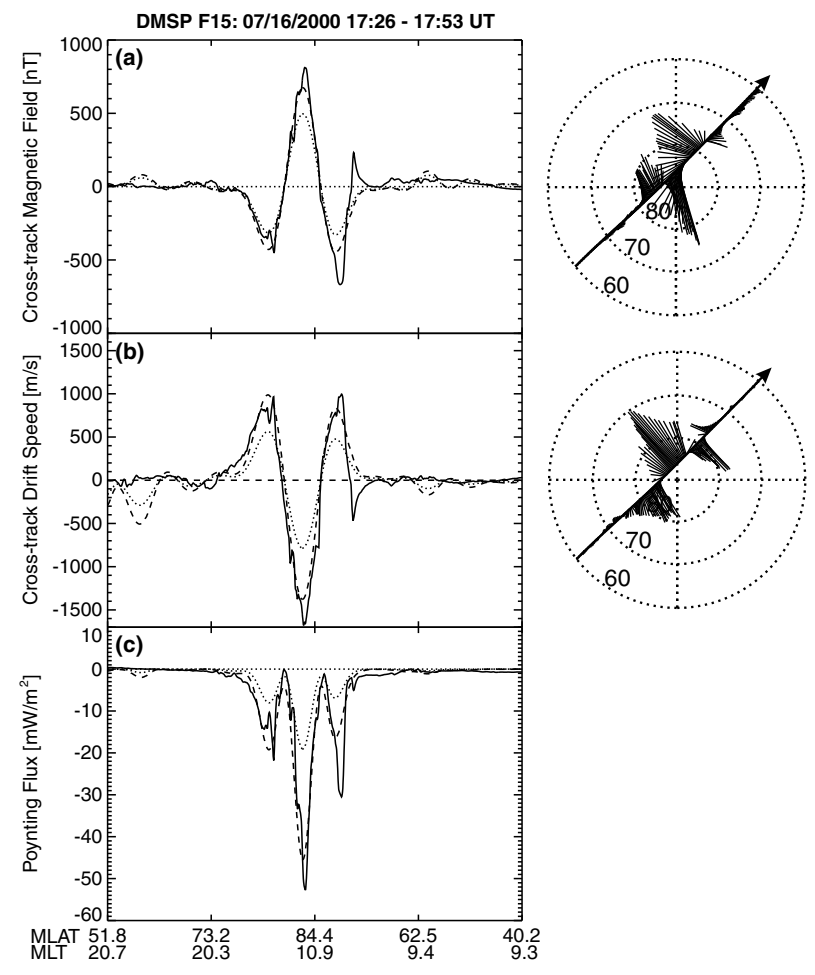

Fig. 6. Comparison of (a) the cross-track magnetic perturbation, (b) the drift speed, and (c) the radial Poynting vector measured by DMSP F15 (solid lines) with calculations using both the unscaled (dotted lines) and scaled (dashed lines) Iridium fit along the DMSP F15 trajectory. The polar plots show the respective vector quantities in AACGM MLT-MLAT coordinates.

can mean that the actual maximum perturbations are not captured in the original Iridium data used as input to the fit. The degree of underestimation depends on how localized the true magnetic field features are and on the spacing of the samples. Previous comparisons between observations by the DMSP and Ørsted satellites and Iridium fit results have shown peak magnetic perturbations observed by DMSP and Ørsted, which are up to a factor of two higher than obtained from the Iridium analysis (Korth et al., 2004). Because $b^{2}$ appears in Eq. (5) one must correct the Iridium fits for this discrepancy to obtain realistic estimates for the radial Poynting vector.

The solid traces in Figs. 6 and 7 show the DMSP F13 and F15 cross-track magnetic field, cross-track plasma drift, and radial Poynting vector, respectively. The polar plots on the right hand sides of these figures show polar projections of the vector magnetic field and plasma drift, where local noon and dusk are at the top and to the left, respectively. The dotted curves show the corresponding quantity evaluated in the Iridium fit along the DMSP satellite tracks. The dashed curves show the Iridium fit results after applying correction factors. Considering first the magnetic perturbations, Fig. 6a shows the cross-track component of the magnetic field perturbation along the DMSP F15 orbit between 17:26 UT and 17:53 UT on 16 July 2000 (solid line). The DMSP F15 satellite is in an

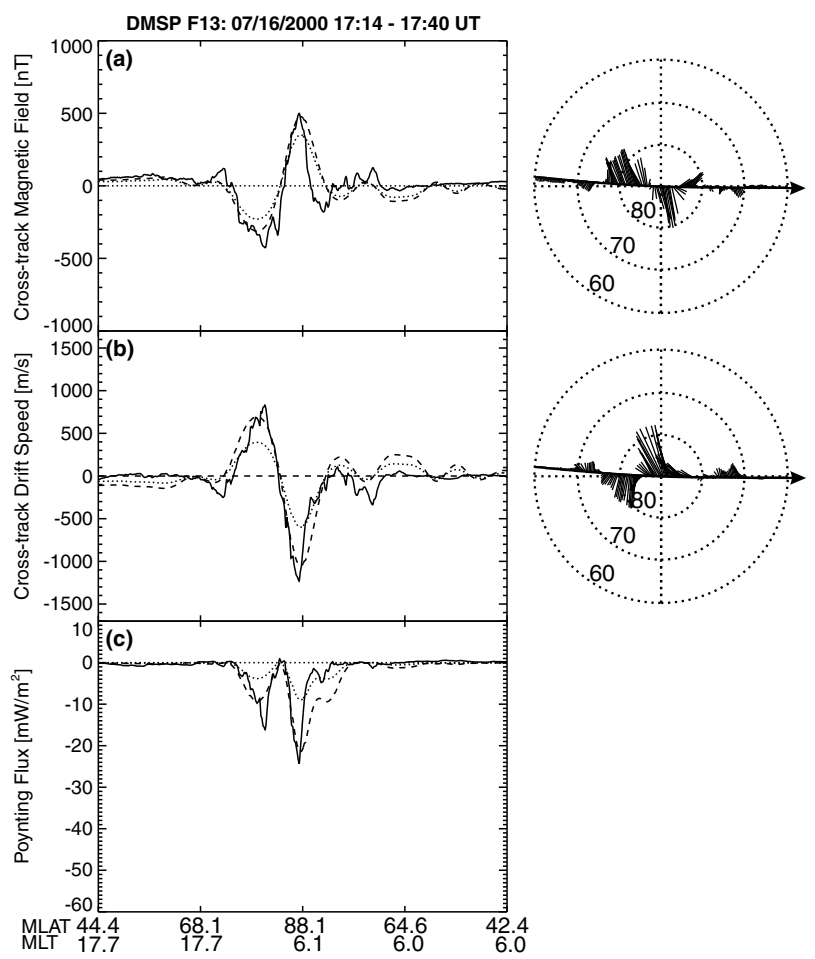

Fig. 7. Comparison of (a) the cross-track magnetic perturbation, (b) the drift speed, and (c) the radial Poynting vector measured by DMSP F13 (solid lines) with calculations using both the unscaled (dotted lines) and scaled (dashed lines) Iridium fit along the DMSP F13 trajectory. The polar plots show the respective vector quantities in AACGM MLT-MLAT coordinates.

approximately 21 MLT to 9 MLT sun-synchronous orbit and the cross-track magnetic field is in the nadir cross spacecraft velocity direction, to the right when standing upright viewing forward. Consistent with the Iridium observations, the DMSP F15 satellite observes a w-shaped perturbation of the magnetic field at high latitudes indicative of the NBZ currents (Iijima et al., 1984; Potemra et al., 1984; Zanetti et al., 1990). The Iridium fit along the DMSP F15 trajectory, shown as a dotted line in Fig. 6a, tracks the F15 measurements, giving reversals at the same locations. Comparison of the F15 polar projection plot with the Iridium currents and $\boldsymbol{b}$ map (Figs. $2 \mathrm{~b}$ and c shows that F15 passes through both currents and observes rotations in the magnetic field consistent with the Iridium results.

The comparison with F13 gives similar results. The DMSP F13 satellite traverses through the polar region in an 18 MLT to 6 MLT sun-synchronous orbit. The cross-track magnetic field component measured by DMSP F13 (Fig. 7a) is in qualitative agreement with the DMSP F15 observations (Fig. 6a), although the w-feature is not quite as pronounced. On the duskside, DMSP F13 measures the clockwise rotation of the magnetic field associated with the downward NBZ current. On the dawnside however, the spacecraft trajectory only skims the tailward edge of the NBZ upward current region (cf. Fig. 2c), where the associated magnetic perturbations are 
both weaker and oriented predominantly parallel to the satellite track. The DMSP comparisons give additional confirmation of the location of the currents inferred from the Iridium results.

As seen in Figs. 6a and 7a, the amplitude in Iridium $\boldsymbol{b}$ is systematically low relative to both F13 and F15. To quantify this underestimate we calculate the integral of the absolute values of the magnetic perturbations from the Iridium fit and DMSP to define a $\boldsymbol{b}$ scaling ratio

$c_{\mathrm{b}}=\frac{\int\left|b_{\mathrm{c}, \mathrm{DMSP}}\right| d S}{\int\left|b_{\mathrm{c}, \text { Iridium }}\right| d S}$.

This gives $c_{\mathrm{b}}=1.38$ for $\mathrm{F} 15$ and $c_{\mathrm{b}}=1.34$ for $\mathrm{F} 13$, so to correct the Iridium $\boldsymbol{b}$ we multiply the Iridium fit results by the average, 1.36. The scaled Iridium results are shown as dashed lines in Figs. 6a and 7a and are in good agreement with both F13 and F15. The small-scale westward perturbation observed by F15 on the dawn side near $72^{\circ}$ MLAT is too narrow for the Iridium analysis to resolve, but, as discussed below, may be significant since it may indicate a dynamo acting at lower latitudes than the high-latitude reconnection convection.

\subsection{DMSP comparison: Plasma drift}

Turning now to the cross-track plasma drift observations, Figs. $6 \mathrm{~b}$ and $7 \mathrm{~b}$ show the cross-track component (solid line) and to the right the vector plot of the $\boldsymbol{E} \times \boldsymbol{B}$ drift speed measurements from the DMSP F13 and F15 drift meter instruments. Both F13 and F15 show strong sunward drift between the NBZ currents with return flows on the equatorward sides of the currents, consistent with convection due to high-latitude reconnection. Moreover, the drifts are very nearly proportional to the negative of the magnetic perturbations suggesting that a simple scaling between $\boldsymbol{b}$ and $\boldsymbol{E}$ exists for this event, as expected for relatively high solar EUV illumination. To compare with the electric field estimated from Iridium, we evaluate the cross-track drift from the Iridium vector fit $\boldsymbol{b}$ and the ionospheric conductivity model using

$\boldsymbol{v}=-\frac{\boldsymbol{b}}{\mu_{0} \Sigma_{\mathrm{P}} B_{0}}$,

where $B_{0}$ is the magnitude of the geomagnetic field. The results from Eq. (7) along the DMSP tracks are shown as dotted lines in Fig. $6 \mathrm{~b}$ and Fig. 7b. The trends and locations of the reversals in the cross-track drift speed obtained from Iridium agree with the F13 and F15 observations.

The drift speeds estimated from the Iridium fits are systematically lower than measured by DMSP. The difference arises in part because the magnetic perturbations are low by the factor $c_{\mathrm{b}}$, but it is also affected by possible systematic differences between the true $\Sigma_{\mathrm{P}}$ and the model. As with $c_{\mathrm{b}}$ we use integrals of the absolute values of $v_{\mathrm{c}}$ and $b_{\mathrm{c}}$ to determine the required factor to scale the modelled conductivity, $c_{\Sigma}$. Specifically we calculate:

$c_{\Sigma}=\left(\frac{\int\left|b_{\mathrm{c}, \text { DMSP }}\right| d S}{\int\left|b_{\mathrm{c}, \text { Iridium }}\right| d S}\right) /\left(\frac{\int\left|v_{\mathrm{c}, \text { DMSP }}\right| d S}{\int\left|v_{\mathrm{c}, \text { Iridium }}\right| d S}\right)=\frac{\Sigma_{\mathrm{P}, \text { true }}}{\Sigma_{\mathrm{P}, \text { model }}}$.
The ratio, DMSP/Iridium, of the cross-track drift integrals is 1.89 for F15 and 1.62 for F13, both larger than $c_{\mathrm{b}}$, indicating that the model conductance is somewhat too high. We then have $c_{\Sigma}=0.73$ for F15 and 0.83 for F13, giving an average correction factor for the conductance of 0.78 . This is a fairly small correction given the use of the F10.7 index as proxy for EUV flux, and we conclude that the Rasmussen et al. model scaled by $c_{\Sigma}$ provides a reasonable description of the conductance distribution for this event. The dashed lines in Figs. $6 \mathrm{~b}$ and $7 \mathrm{~b}$ show the results for the cross-track drift when both the magnetic perturbation and conductivity correction factors are used.

\subsection{Electromagnetic energy flux results}

The Poynting vector results from DMSP F13 and F15 calculated using Eq. (1) together with the estimates from Iridium and the conductance are shown in Fig. 6c and Fig. 7c. Negative values represent downward energy flux. Uncorrected Iridium estimates are shown by the dotted lines, while the dashed lines give the results corrected using both $c_{\mathrm{b}}$ and $c_{\Sigma}$. The DMSP observations show energy flux flowing almost exclusively into the ionosphere, consistent with previous observations and supporting the approximation of a universally downward-directed Poynting vector, see Eq. (5). The Iridium-derived, scaled Poynting vector agrees in location with the DMSP results quite well, with the exception of the F13 case equatorward of the pole on the morning side, where the Iridium based estimate is $\sim 10 \mathrm{~mW} / \mathrm{m}^{2}$ while the F13 observation is less than $3 \mathrm{~mW} / \mathrm{m}^{2}$. The integrals of the energy flux from DMSP and the scaled Iridium estimate agree on average though the Iridium estimates give peaks that are somewhat lower than F15 and the above mentioned region of $10 \mathrm{~mW} / \mathrm{m}^{2}$ flux dawnward of the flux observed by F13. Otherwise the Iridium estimated fluxes reproduce the DMSP results reasonably well.

In Fig. 8, we present the global distribution of the electromagnetic energy flux on 16 July 2000, from 17:00 UT to 18:00 UT, determined from Eq. (5) using the corrections derived above. The figure demonstrates that during this interval of northward IMF the bulk of the electromagnetic energy enters the ionosphere poleward of $78^{\circ}$ MLAT with a prominent peak near $87^{\circ}$ MLAT in the pre-noon region, where the maximum flux is near $50 \mathrm{~mW} / \mathrm{m}^{2}$. Comparison of the global radial Poynting vector distributions with the DMSP trajectory data, which are overlaid on Fig. 8, show that the F15 satellite passed closer to the maximum of the electromagnetic energy deposition than the F13 satellite, which is consistent with the relative magnitudes of the radial Poynting vector derived from the two DMSP spacecraft. The magnitude of the energy flux peaks at $48 \mathrm{~mW} / \mathrm{m}^{2}$ in this region, while the maximum energy flux from DMSP F15 is $52 \mathrm{~mW} / \mathrm{m}^{2}$. The total electromagnetic energy input poleward of $75^{\circ}$ MLAT is estimated to be $51 \mathrm{GW}$. If we somewhat arbitrarily take the uncertainty to be twice the difference in the scaling factors between the two DMSP satellites, $\pm 2 \%$ for $c_{\mathrm{b}}$ and $\pm 6 \%$ for 


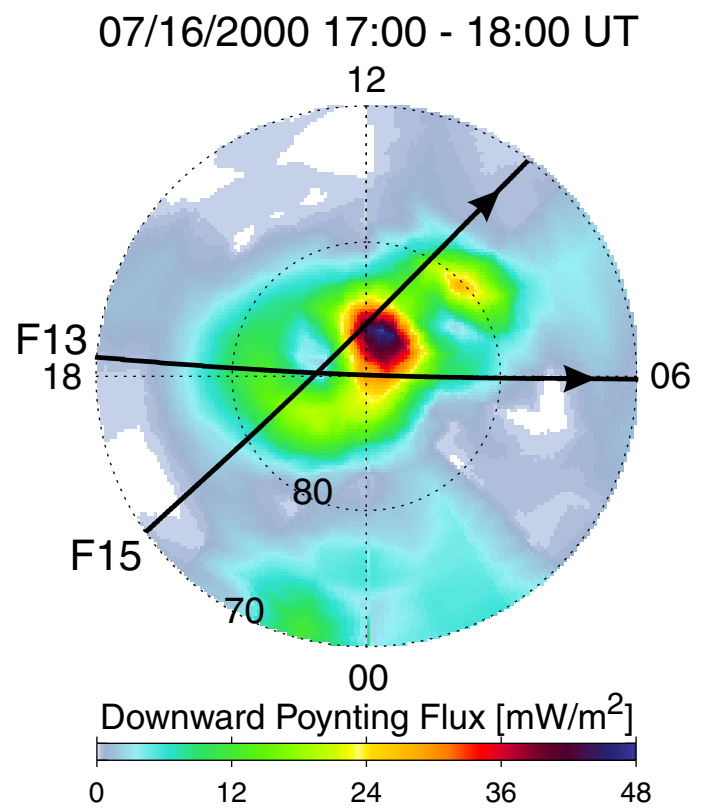

Fig. 8. The global distribution of the Iridium determined radial Poynting vector on 16 July 2000, from 17:00 UT to 18:00 UT in AACGM MLT-MLAT coordinates.

$c_{\Sigma}$, we estimate that the total energy input using Eq. (5) is uncertain to $\pm 20 \%$.

\section{Comparison with precipitating particle energy flux}

The auroral images in Fig. 4 show a localized region of enhanced emissions in the region of upward current. As discussed above, the SI12 spectrograph of the FUV instrument, which is sensitive to proton-induced auroral emissions, does not record emissions above background during the event. In addition, the DMSP F15 SSJ/4 instrument (Hardy et al., 1984) measures enhanced accelerated electron precipitation in the region of upward current. The coincidence of the emissions with upward current, accelerated electrons, and the absence of significant proton precipitation signatures suggest that the emissions are predominantly caused by precipitating electrons.

We then estimate the electron flux from the FUV image results as follows. The conversion of the WIC count rate to electron energy flux is governed by the energy dependent transfer function from emission intensity to energy flux (Frey et al., 2003). Since the spectrum of the precipitating population is not determined by the WIC data, we use the DMSP F15 SSJ/4 particle data during the satellite's transit through the enhanced emission region to determine a characteristic electron energy. The characteristic electron energy for the DMSP F15 pass from 17:26 UT to 17:53 UT on 16 July 2000, in the pre-noon region poleward of $80^{\circ}$ MLAT, where the intensity of the emissions peaks, is $\sim 750 \mathrm{eV}$. The appropriate conversion factor at this energy is $2 \cdot 10^{-3} \mathrm{~mW} / \mathrm{m}^{2} /$ count (Frey et al., 2003) and the corresponding electron energy flux

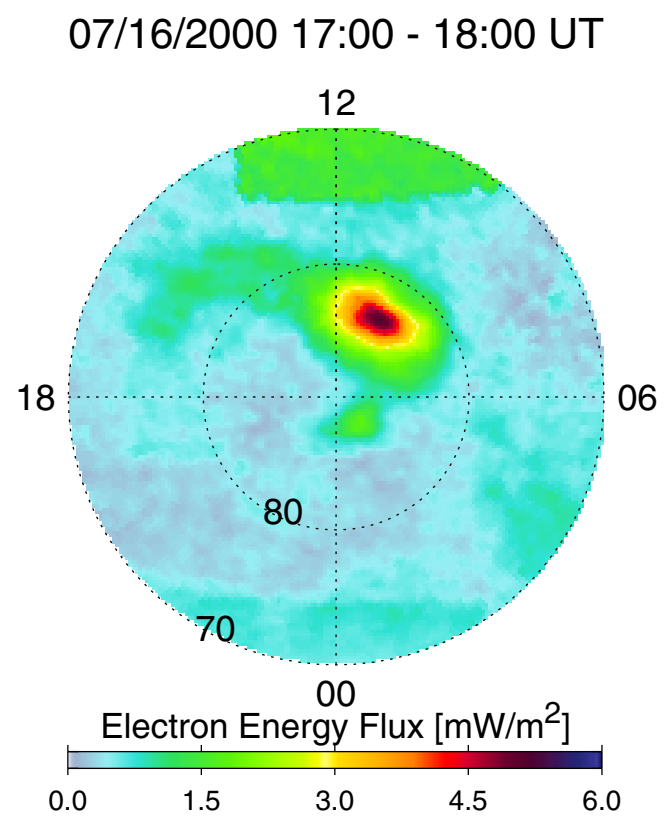

Fig. 9. The global distribution of the precipitating electron energy flux on 16 July 2000, from 17:00 UT to 18:00 UT in AACGM MLTMLAT coordinates.

for the 17:00-18:00 UT interval is presented in Fig. 9. The bulk of the energy flux enters the ionosphere in the highlatitude pre-noon region, where the upward field-aligned currents are located. While the maximum electromagnetic energy flux is observed where the magnetic perturbations maximize, the electron precipitation energy flux is largest where the upward Birkeland currents are strongest. For this reason, the electron energy flux and electromagnetic energy flux are not coincident but complementary. The peak precipitating energy flux is $5 \mathrm{~mW} / \mathrm{m}^{2}$, and the total energy precipitating poleward of $75^{\circ}$ MLAT is $6 \mathrm{GW}$. The electromagnetic energy input during the event is eight times larger than the particle energy flux. The auroral emissions thus represent a modest fraction of the overall energy transport between the magnetosphere and the ionosphere for this event.

\section{Relative importance of reconnection and viscous dy- namos}

In the anti-parallel merging paradigm, northward IMF leads to high-latitude reconnection, poleward of the cusp, and reversed convection at high latitudes is a distinctive characteristic of lobe reconnection (Russell, 1972; Maezawa, 1976). The sunward convection in the polar cap with return flows at lower latitudes as observed here by the DMSP satellites and the corresponding polarity of the NBZ Birkeland currents confirm that this process persists for several hours during this event. With the shift of the reconnection site to latitudes poleward of the cusp, the reconnection and viscous dynamos should be spatially separated. The viscous dynamo is believed to be primarily driven by the KH instability driven by 


\section{7/16/2000 16:00 - 19:00 UT}

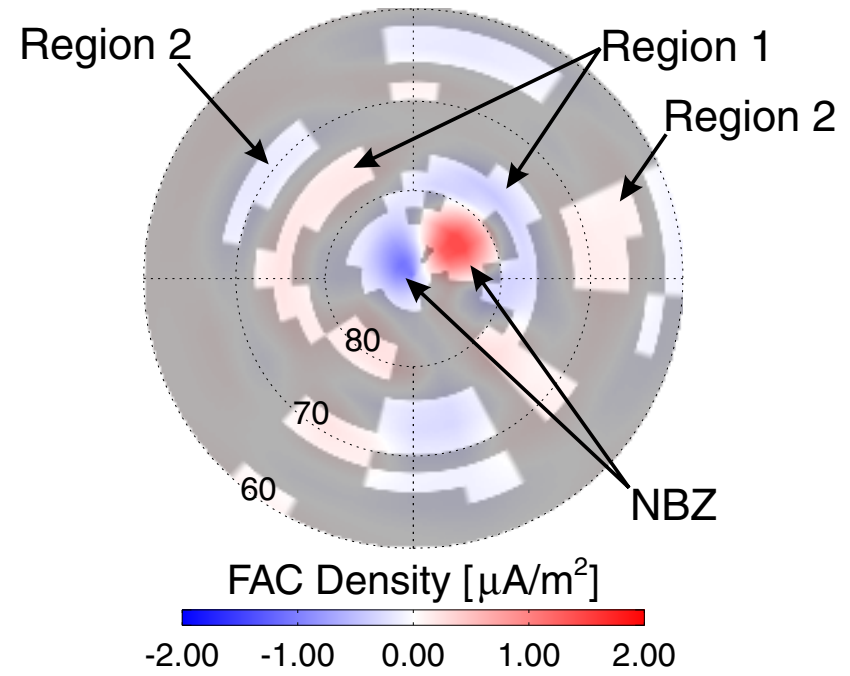

Fig. 10. Average Birkeland current distribution on 16 July 2000, from 16:00 UT to 19:00 UT in AACGM MLT-MLAT coordinates obtained from three non-overlapping hourly snapshots. Upward flowing Birkeland currents are shown in red, blue colors represent currents in the downward direction. Birkeland currents below the quiet-time baseline are masked in gray.

shear flows between the solar wind and magnetospheric plasmas at the magnetopause (e.g., Rostoker et al., 1987). For a simplified model of a tangential discontinuity in an incompressible magnetized plasma, the condition for instability of a wave propagating in direction $\hat{\boldsymbol{k}}$ is given by (e.g., Landau and Lifschitz, 1960; Kivelson and Chen, 1995):

$\left[\hat{\boldsymbol{k}} \cdot\left(\boldsymbol{u}_{\mathbf{2}}-\boldsymbol{u}_{\mathbf{1}}\right)\right]^{2}>\frac{\rho_{1}+\rho_{2}}{\mu_{0} \rho_{1} \rho_{2}}\left[\left(\boldsymbol{B}_{\mathbf{1}} \cdot \hat{\boldsymbol{k}}\right)^{2}+\left(\boldsymbol{B}_{\mathbf{2}} \cdot \hat{\boldsymbol{k}}\right)^{2}\right]$,

where $\boldsymbol{u}$ and $\rho$ are the plasma velocity and density, respectively, $\boldsymbol{B}$ is the magnetic field, and the indices 1 and 2 represent the two sides of the discontinuity. Assuming that the KH waves propagate anti-sunward, it can be seen from Eq. 9) that the dot products on the right hand side vanish for purely northward or southward IMF, when the draped magnetosheath field at the flanks has no component in the flow direction. This corresponds to minimum stabilization of the instability by the magnetic field tension. The occurrence of $\mathrm{KH}$ is therefore most likely during these conditions so the viscous dynamo should be particularly strong. The combination of factors during northward IMF, poleward displacement of reconnection flows and optimal conditions for $\mathrm{KH}$ instability, make the conditions of this event favorable for identifying the viscous-driven dynamo.

\subsection{Viscous driven currents}

The reconnection and viscous interactions drive plasma circulation in the magnetosphere corresponding to ionospheric convection cells. The plasma flows are associated with the electric fields and currents, which in the Northern Hemisphere ionosphere are directed toward and away from the center of the convection cell for clockwise and counterclockwise circulation, respectively. Field-aligned currents are present within each convection vortex and often also at the equatorward edge corresponding to shielding. In the Northern Hemisphere, currents converging in clockwise convection cells are diverted as upward Birkeland currents, while diverging currents in counter-clockwise cells are fed by downward oriented currents.

To bring out low intensity current systems we averaged the Birkeland current patterns from the three one-hour intervals from 16:00 UT to 19:00 UT, and the result is shown in Fig. 10 in the same format as used in Fig. 2c. The averaged Iridium observations show the presence of two and possibly three large-scale Birkeland current systems. Most prominent in Fig. 10 are the high-latitude NBZ currents, measuring $0.85 \mathrm{MA}$ and 0.76 MA for the upward and downward current, respectively. The NBZ currents are associated with reversed lobe cell convection driven by reconnection. Equatorward of the NBZ currents is a pair of Region-1 sense currents, which integrate to 0.52 MA and 0.40 MA on the dawnside and duskside, respectively. The Region-1 sense currents are the right sense to be driven by a viscous interaction. However, the Region-1 sense currents may also facilitate closure of the NBZ system, much as Region 2 facilitates closure of Region 1 for southward IMF. Thus, the Region-1 sense currents cannot be associated unambiguously with either the reconnection or viscous dynamo. On the other hand, Region2 currents accomplish shielding of the inner magnetosphere from the dynamo that drives Region 1, in this case the viscous interaction. The presence of Region 2 might therefore be taken as an indication for the action of a viscous dynamo. The signatures of the Region-2 Birkeland currents are well organized in Fig. 10, although about half of the regions featuring the Region- 2 sense currents are below the noise level. It is important to consider the intensities of the NBZ, Region1 sense, and Region- 2 sense currents relative to the baseline noise. Referring to Fig. 3, we see that the NBZ currents at co-latitudes between $0^{\circ}$ and $10^{\circ}$ are well in excess of the noise level and that the Region- 1 sense currents at colatitudes from $10^{\circ}$ to $15^{\circ}$, although not as prominent, are above the baseline noise. The Region- 2 currents, near $20^{\circ}$ co-latitude, have an average current density within a standard deviation of the baseline noise, so their identification is not as firm as the other currents. With this in mind, the total Region-2 Birkeland currents are 0.19 MA and 0.16 MA on the dawnside and duskside, respectively, which we interpret as upper limits for the Region- 2 currents that could be driven by a viscous dynamo.

\subsection{Large scale electric potential pattern}

One can also examine the electric potential structure implied by the currents. Given the success in reproducing the cross-track DMSP plasma drift, we have some confidence in Eq. (3) relating the magnetic perturbations to the electric 


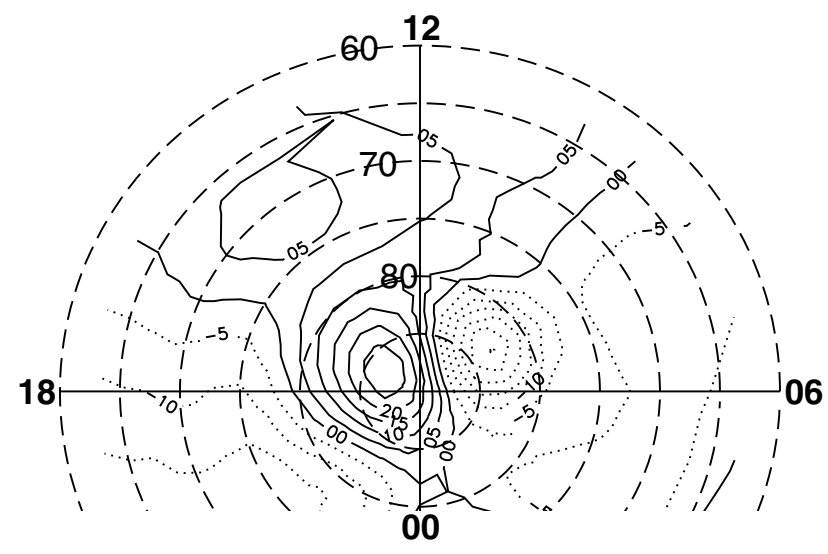

Fig. 11. Iridium electric potential pattern on 16 July 2000, 17:00 UT to 18:00 UT in AACGM MLT-MLAT coordinates.

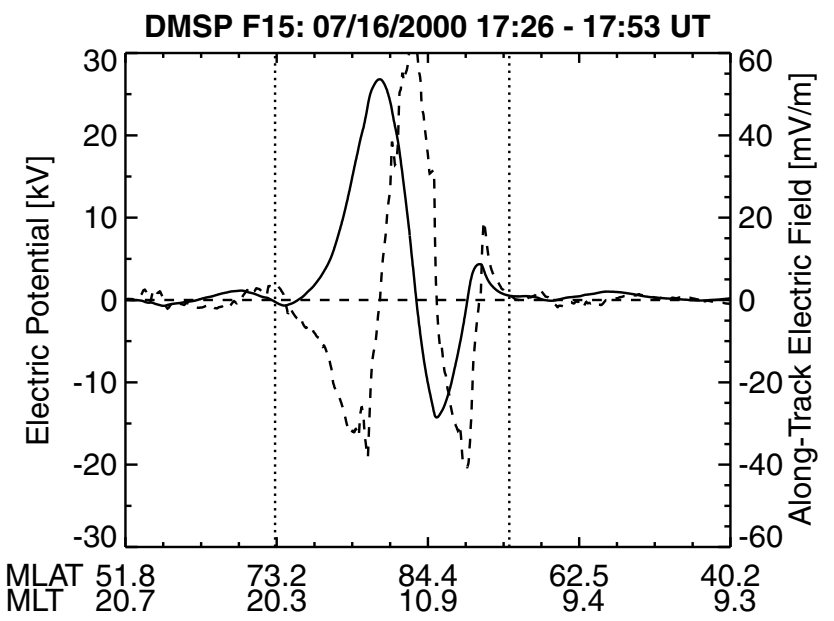

Fig. 12. Electric potential (solid line) derived from the along-track electric field component (dashed line) for the DMSP F15 pass. The vertical dashed lines mark the transition between Region-1 and Region-2 sense Birkeland currents.

field via the conductivity. The approximation appears to hold in regions of significant drift observed by DMSP and can be expected also to hold sunward of that but not on the nightside, where conductivity gradients could be large and the conductance model does not apply. From the average magnetic perturbations corresponding to the currents of Fig. 10 and using the correction factors for the magnetic perturbations and the conductivity obtained above, we derive electric field estimates using Eq. (3) and from that calculate a potential pattern. The result is shown in Fig. 11 for the region where the estimate is probably valid. The positive/negative potential pair associated with the NBZ system is clear, and we obtain peak potentials of about $+25 \mathrm{kV}$ and $-30 \mathrm{kV}$. The signature of a viscous dynamo would be additional potential maxima, positive in the morning and negative in the evening corresponding to the Region-1 sense currents of Fig. 10. Figure 11 does not show such a potential structure in the $70^{\circ}$ to $75^{\circ}$ latitude range where the Region- 1 sense currents flow.

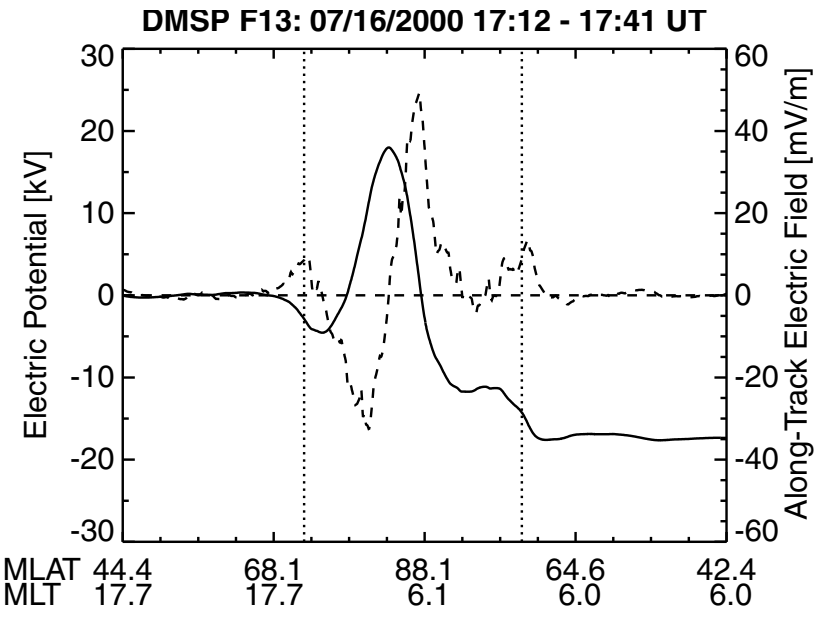

Fig. 13. Electric potential (solid line) derived from the along-track electric field component (dashed line) for the DMSP F13 pass. The vertical dashed lines mark the transition between Region- 1 and Region-2 sense Birkeland currents.

Neither does it show characteristic potentials associated with the Region-2 sense currents. There are other potential signatures of about $5 \mathrm{kV}$ to $10 \mathrm{kV}$ that do not correlate with any organized Birkeland currents, indicating that they are due to the integrated effects at the noise level of the Iridium fit results. We conclude that the Iridium observations alone provide no definitive confirmation of a viscous dynamo and indicate an upper limit for a viscous driven potential of $5 \mathrm{kV}$ to $10 \mathrm{kV}$.

\subsection{Smaller scale electric potentials}

The DMSP drift and magnetometer data displayed signatures on spatial scales smaller than the Iridium fits could resolve, so we now turn to these data in search of viscous cell signatures. Figures 6 and 7 for F15 and F13, respectively, show small magnetic perturbations and drift signatures equatorward of the primary NBZ signatures. For F15 there is a narrow region with a $200 \mathrm{nT}$ cross-track perturbation and $500 \mathrm{~m} / \mathrm{s}$ drift at about $76^{\circ}$ MLAT in the morning sector. The F13 data show smaller features, $100 \mathrm{nT}$ and $200 \mathrm{~m} / \mathrm{s}$, in the $70^{\circ}$ to $75^{\circ}$ MLAT range at both dawn and dusk. The polarities of these signatures are consistent with viscous-driven convection. To quantify the potential associated with these signatures, we integrate the along track electric field for both F13 and F15. Figures 12 and 13 show the electric potential (solid line) derived from the along-track component of the electric field (dotted line) for each of the DMSP passes shown in Figs. 6 and 7. Both F13 and F15 pass through the positive NBZ potential in the afternoon and observe maximum potentials of about $+18 \mathrm{kV}$ and $+27 \mathrm{kV}$, respectively. F15 also passes through the morning potential and observes a negative potential of $-15 \mathrm{kV}$. F13 however does not pass through the morning convection cell. There appears to be a systematic baseline drift in the F13 data leading to the large accumulated potential of $-35 \mathrm{kV}$ at the end of the track. This level shift issue is not uncommon in analyses of these data 
and is often resolved by adjusting the baseline, but for our purposes this is not necessary. This artifact is not present to a significant degree in the F15 data. Not surprisingly, the potential drops associated with the NBZ convection observed by the DMSP satellites are consistent with the Iridium derived potentials, owing to the fact that the Iridium calculation is scaled to agree with the DMSP plasma drifts.

The features of interest with respect to the viscous interaction are the smaller potential shifts equatorward of the NBZ convection. In F15, the narrow convection region on the morning side is associated with a $5 \mathrm{kV}$ potential. In F13, the low level equatorward drift regions at dawn and dusk are each associated with potential drops of $5 \mathrm{kV}$. These structures are close to or below the uncertainty in the Iridium estimate. The F13 features would be resolvable in the Iridium inversions, but their amplitudes are too small to be clearly distinguished from the background noise. In the case of F15 the feature is too narrow to be resolved in the Iridium inversions. The DMSP data therefore indicate the presence of a viscous interaction potential of about $5 \mathrm{kV}$. The viscous-sense electric fields fall in the latitude range between the Region- 1 and Region-2 sense currents consistent with this interpretation. Since the solar wind and IMF conditions for this event are highly favorable to the $\mathrm{KH}$ instability at the magnetopause flanks, we would expect that the viscous-driven potential is usually lower than this.

\section{Discussion and summary}

We examined an interval of stable northward IMF on 16 July 2000, to assess the relative contribution of electromagnetic and particle energy flux to the total energy exchange between the magnetosphere and the ionosphere under these conditions. The global distribution of the radial Poynting vector was derived from magnetic field perturbations measured by the constellation of Iridium satellites and a model for the ionospheric conductivity. Quantitative comparison with DMSP magnetometer and drift meter data were used to derive corrections to both the Iridium fit results and the conductance model. Particle energy flux was calculated from the intensity of far-ultraviolet auroral emissions measured by IMAGE FUV/WIC under the assumption that the incident particle population consists solely of electrons precipitating at the characteristic peak energy obtained from DMSP observations. We found that the electromagnetic energy input into the ionosphere poleward of $75^{\circ}$ MLAT is $51 \mathrm{GW}$ while that from electron precipitation is $6 \mathrm{GW}$, about eight times smaller. We note that this difference is twice the factor of four estimated by Ahn et al. (1983). Particle precipitation therefore represents a fairly small fraction of the overall ionospheric energy deposition for this event.

The results for the energy flux are remarkable in several respects. First, the $57 \mathrm{GW}$ total energy deposition is higher than one would have expected. It is comparable to the average heating power at auroral latitudes of $84 \mathrm{GW}$ from the EISCAT and SABRE radars (Kosch and Nielsen, 1995) and is also in the range of heating rates obtained by other techniques for non-storm time moderate activity which are typically 50 to $100 \mathrm{GW}$ (e.g., Lu et al., 1996; Buonsanto et al., 1999; Slinker et al., 1999). Foster et al. (1983) conducted a statistical analysis of AE-C satellite measurements, binning ion drift and particle precipitation as a function of $K_{p}$. They obtained $35 \mathrm{GW}$ for the $K_{p}$ range from 0 to 3, corresponding to this event for which $K_{p}$ was between 2 and 3 and the quick look $\mathrm{AE}$ was below $100 \mathrm{nT}$ throughout. In addition, the statistical analysis of Ahn et al. (1983) based on ground magnetometer data yields less than $25 \mathrm{GW}$ total energy input for an AE index of $100 \mathrm{nT}$. Thus, despite the strongly northward IMF conditions, the total energy flux is higher than one would expect for these conditions and comparable to that occurring during moderate activity.

Moreover, the concentration of the energy deposition poleward of $78^{\circ}$ MLAT implies that the energy flux is unusually high. Typical maximum energy fluxes are in the range 10 to $20 \mathrm{~mW} / \mathrm{m}^{2}$ (Thayer, 1998b, 2000; Lu et al., 1996; Buonsanto et al., 1999; Slinker et al., 1999). For this event however, the maximum electromagnetic energy flux was $50 \mathrm{~mW} / \mathrm{m}^{2}$, considerably higher than typical maxima inferred from radars and assimilation techniques. It is also at the high end of energy fluxes observed from satellites which were observed at auroral latitudes (Kelley et al., 1991; Gary et al., 1995). Our result implies that the localized heating near the magnetic pole during this event was particularly strong and should be associated with a strong localized thermospheric temperature enhancement and corresponding upwelling comparable in intensity to that occurring at auroral latitudes during active times. That the localized heating can be this intense during northward IMF is not anticipated and needs to be included in analyses of the thermosphere/ionosphere response to solar wind forcing.

We suggest that the localization of the current systems and particle precipitation poleward of $78^{\circ}$ MLAT on the dayside during this event is an important reason that the results we obtain for the total energy and peak energy flux density are larger than previous results would lead one to expect. Studies based primarily on ground magnetometer data from the Northern Hemisphere do not have extensive coverage in the region where the ionospheric currents were flowing during this event and would therefore yield underestimates of the currents under these conditions (e.g., Ahn et al., 1983; Lu et al., 1996). Similarly, studies based on radar observations would underestimate the energy transport if their fields of view did not include the region very close to the pole (e.g., Fujii et al., 1999; Thayer, 2000). Statistical studies using satellite data (e.g., Foster et al., 1983; Gary et al., 1995) will also tend to underestimate the energy transport during strongly northward IMF because the satellite tracks intercept the comparatively small region of convection on only a fraction of the orbits. The evolution of signatures from the DMSP F15 satellite for this case illustrates how this could happen. For this event the DMSP F15 satellite did not observe significant magnetic or electric fields following 06:00 UT until after 15:00 UT even though the Iridium key 
parameter data (cf. Anderson et al., 2002) show that the magnetic perturbations were present throughout this time from $75^{\circ}$ MLAT to the pole and were in fact stronger than during the interval analyzed here. If one based analysis on F15 only one would not have detected energy transport prior to 15:00 UT even though it appears to have been more intense than that reported here.

The strongly northward IMF conditions allowed us to provide a new upper limit for the contribution of the viscous interaction to solar wind forcing. Sunward convection in the dayside polar region consistent with reconnection poleward of the cusp was clear throughout the event. The high-latitude reconnection convection was therefore spatially separated from the viscous convection at lower latitudes, which allowed unambiguous estimation of the contributions of each. We found that the average magnitude of the reconnectiondriven NBZ Birkeland currents is with $0.81 \mathrm{MA}$ at least a factor of five larger than the Region- 2 currents $(<0.17 \mathrm{MA})$, presumably driven by the viscous interaction. The potential drop associated with high-latitude reconnection is about $45 \mathrm{kV}$, while that attributable to a viscous interaction is at most $\sim 5 \mathrm{kV}$. This limit is at the low end of previous viscous dynamo estimates. Reiff et al. (1981) report a significant $(35 \mathrm{kV})$ persistent potential drop due to viscous interaction, while Mozer (1984) attributes at most $10 \%$ (5 to $15 \mathrm{kV})$ of the magnetospheric electric potential to viscous interaction, consistent with our result. Wygant et al. (1983) demonstrate that the contributions to the polar cap potential not associated with the reconnection process can be limited to less than $20 \mathrm{kV}$. Our result substantiates the results of Mozer (1984) and Wygant et al. (1983) in particular because for this event the KH instability should have been particularly favored and the evidence of the viscous interaction should have been particularly clear. It has been suggested that for southward IMF the $\mathrm{KH}$ interaction may promote additional reconnection in flow vortices where anti-parallel fields are wrapped up in close proximity (Nykyri and Otto, 2001), a result that is not addressed here. We conclude that the viscous interaction is unlikely to be a significant contributor during northward IMF conditions. The possibility remains that viscous effects may promote the reconnection dynamo for southward IMF conditions.

Acknowledgements. We thank Iridium Satellite LLC for providing the engineering magnetometer data for scientific analysis. Support for processing and analysis of the Iridium magnetometer data was provided by NSF under grants ATM-9819804, ATM-0101064, and ATM-0334668 and by NASA under grants NAG5-10849 and NAG5-12303. Any opinions, findings, and conclusions or recommendations expressed in this material are those of the author(s) and do not necessarily reflect the views of the National Science Foundation. We thank the ACE team for the use of the MAG and SWEPAM data made available via the ACE Level 2 data base and acknowledge the Center for Space Sciences at the University of Texas at Dallas and the US Air Force for providing the DMSP thermal plasma data. F. Rich provided the DMSP magnetometer data. Finally, we thank D. Hardy, F. Rich, and P. Newell for providing the DMSP particle data.
Topical Editor T. Pulkkinen thanks L. Blomberg and another referee for their help in evaluating this paper.

\section{References}

Ahn, B.-H., Akasofu, A.-I., and Kamide, Y.: The joule heat production rate and the particle energy injection rate as a function of the geomagnetic indices AE and AL, J. Geophys. Res., 88, 6275-6287, 1983.

Anderson, B. J., Takahashi, K., and Toth, B. A.: Sensing global Birkeland currents with Iridium engineering magnetometer data, Geophys. Res. Lett., 27, 4045-4048, 2000.

Anderson, B. J., Takahashi, K., Kamei, T., Waters, C. L., and Toth, B. A.: Birkeland currents sytem key parameters derived from Iridium observations: Method and initial validation results, J. Geophys. Res., 107, 2002.

Aulanier, G., DeLuca, E. E., Antiochos, S. K., McMullen, R. A., and Golub, L.: The Topology and Evolution of the Bastille Day flare, Astrophys. J., 540, 1126-1142, 2000.

Axford, W. I. and Hines, C. O.: A Unifying Theory of HighLatitude Geophysical Phenomena and Geomagnetic Storms, Can. J. Phys., 39, 1443-1464, 1961.

Baker, K. B. and Wing, S.: A new magnetic coordinate system for conjugate studies at high latitudes, J. Geophys. Res., 94, 91399143, 1989.

Buonsanto, M. J., Gonzalez, S. A., Lu, G., Reinisch, B. W., and Thayer, J. P.: Coordinated incoherent scatter radar study of the January 1997 storm, J. Geophys. Res., 104, 24 625-24 637, 1999.

Cole, K. D.: Joule Heating of the Upper Atmosphere, Aust. J. Phys., 15, 221-235, 1962.

Crooker, N. U.: Dayside Merging and Cusp Geometry, J. Geophys. Res., 84, 951-959, 1979.

Dungey, J. W.: Interplanetary Magnetic Field and the Auroral Zones, Phys. Rev. Lett., 6, 47-48, 1961.

Erlandson, R. E., Zanetti, L. J., Potemra, T. A., Block, L. P., and Holmgren, G.: Viking Magnetic and Electric Field Observations of Pc 1 Waves at High Latitudes, J. Geophys. Res., 95, 59415955, 1990.

Förster, M., Mishin, V. M., Saifudinova, T. I., Bazarshapov, A. D., Shirapov, D. S., and Lunyushkin, S. B.: Contribution of two Processes to Magnetospheric Energy and Momentum Input During the CDAW-W Period, Ann. Geophys., 9, 495-499, 1991.

Foster, J. C., St.-Maurice, J.-P., and Abreu, V. J.: Joule heating at high latitudes, J. Geophys. Res., 88, 4885-4896, 1983.

Frey, H. U., Mende, S. B., Immel, T. J., Gérard, J.-C., Hubert, B., Habraken, S., Spann, J., Gladstone, G. R., Bisikalo, D. V., and Shematovich, V. I.: Summary of Quantitative Interpretation of IMAGE Far Ultraviolet Auroral Data, Space Sci. Rev., 109, 255283, 2003.

Fujii, R., Nozawa, S., Buchert, S. C., and Brekke, A.: Statistical Characteristics of Electromagnetic Energy Transfer Between the Magnetopshere, the Ionosphere, and the Thermosphere, J. Geophys. Res., 104, 2357-2365, 1999.

Gary, J. B., Heelis, R. A., Hanson, W. B., and Slavin, J. A.: FieldAligned Poynting Flux Observations in the High-Latitude Ionosphere, J. Geophys. Res., 99, 11 417-11427, 1994.

Gary, J. B., Heelis, R. A., and Thayer, J. P.: Summary of FieldAligned Poynting Flux Observations From DE 2, Geophys. Res. Lett., 22, 1861-1864, 1995.

Hardy, D. A., Schmitt, L. K., Gussenhoven, M. S., Marshall, F. J., Yeh, H. C., Shumaker, T. L., Hube, A., and Pantazis, J.: Precipi- 
tating electron and ion detectors (SSJ/4) for the block 5D/flights 6-10 DMSP satellites: Calibration and data presentation, Tech. Rep. AFGL-TR-84-0317, Air Force Geophys. Lab., Hanscom Air Force Base, Mass., 1984.

Heikkila, W. J.: Magnetic Reconnection, Merging, and Viscous Interaction in the Magnetosphere, Space Sci. Rev., 53, 1-44, 1990.

Iijima, T. and Shibaji, T.: Global Characteristics of Northward IMFAssociated (NBZ) Field-Aligned Currents, J. Geophys. Res., 92, 2408-2424, 1987.

Iijima, T., Potemra, T. A., Zanetti, L. J., and Bythrow, P. F.: LargeScale Birkeland Currents in the Dayside Polar Region During Strongly Northward IMF: A New Birkeland Current System, J. Geophys. Res., 89, 7441-7452, 1984.

Jordanova, V. K., Thorne, R. M., Farrugia, C. J., Dotan, Y., Fennell, J. F., Thomsen, M. F., Reeves, G. D., and McComas, D. J.: Ring Current Dynamics During the 13-18 July 2000 Storm Period, Sol. Phys., 204, 361-375, 2001.

Keiling, A., Wygant, J. R., Cattell, C. A., Mozer, F. S., and Russell, C. T.: The Global Morphology of Wave Poynting Flux: Powering the Aurora, Science, 299, 383-386, 2003.

Kelley, M. C., Knudsen, D. J., and Vickrey, J. F.: Poynting flux measurements on a satellite: A diagnostic tool for space research, J. Geophys. Res., 96, 201-207, 1991.

Kivelson, M. G. and Chen, S.-H.: The Magnetopause: Surface Waves and Instabilities and Their Possible Dynamical Consequences, In Physics of the Magnetopause, P. Song, B. U. Ö Sonnerup, and M. F. Thomsen, (Eds.), Geophysical Monograph 90, 257-268, American Geophysical Union, Washington, 1995.

Korth, H., Anderson, B. J., Wiltberger, M. J., Lyon, J. G., and Anderson, P. C.: Intercomparison of Ionospheric Electrodynamics From the Iridium Constellation With Global MHD Simulations, J. Geophys. Res., 109, A07 307, 2004.

Kosch, M. J. and Nielsen, E.: Coherent radar estimates of average high-latitude ionospheric Joule heating, J. Geophys. Res., 100, 12 201-12 215, 1995.

Landau, L. D. and Lifschitz, E. M.: Electrodynamics of Continous Media, Pergamon Press, New York, 1960.

Lu, G., Richmond, A. D., Emery, B. A., and Roble, R. G.: Magnetosphere-Ionosphere-Thermosphere Coupling: Effect of Neutral Winds on Energy Transfer and Field-Aligned Current, J. Geophys. Res., 100, 19643-19659, 1995.

Lu, G., Emery, B. A., Roger, A. S., Lester, M., Taylor, J. R., Evans, D. S., Ruohoniemi, J. M., Denig, W. G., de la Beaujardiere, O., Frahm, R. A., Winningham, J. D., and Chenette, D. L.: Highlatitude ionosphere electrodynamics as determined by the assimilative mapping of ionospheric electrodynamics procedure for the conjunctive SUNDIAL/ATLAS 1/GEM period of 28-29 March 1992, J. Geophys. Res., 101, 26 697-26718, 1996.

Lummerzheim, D., Brittnacher, M., Evans, D., Germany, G. A., Parks, G. K., Rees, M. H., and Spann, J. F.: High Time Resolution Study of the Hemispheric Power Carried by Energetic Electrons Into the Ionosphere During the 19-20 May 1996 Auroral Activity, Geophys. Res. Lett., 24, 987-990, 1997.

Maezawa, K.: Magnetospheric Convection Induced by the Positive and Negative Z Components of the Interplanetary Magnetic Field: Quantitative Analysis Using Polar Cap Magnetic Records, J. Geophys. Res., 81, 2289-2303, 1976.

McComas, D. J., Bame, S. J., Barker, P. L., Feldman, W. C., Phillips, J. L., Riley, P., and Griffee, J. W.: Solar Wind Electron Proton Alpha Monitor (SWEPAM) for the Advanced Composition Explorer, Space Sci. Rev., 86, 563-612, 1998.

Mende, S. B., Heetderks, H., Frey, H. U., Lampton, M., Geller,
S. P., Abiad, R., Siegmund, O. H. W., Tremsin, A. S., Spann, J., Dougani, H., Fuselier, S. A., Magoncelli, A. L., Bumala, M. B., Murphree, S., and Trondsen, T.: Far ultraviolet imaging from the IMAGE spacecraft. 2. Wideband FUV imaging, Space Sci. Rev., 91, 271-285, 2000a.

Mende, S. B., Heetderks, H., Frey, H. U., Lampton, M., Geller, S. P., Habraken, S., Renotte, E., Jamar, C., Rochus, P., Spann, J., Fuselier, S. A., Gerard, J.-C., Gladstone, R., Murphree, S., and Cogger, L.: Far ultraviolet imaging from the IMAGE spacecraft. 1. System design, Space Sci. Rev., 91, 243-270, 2000 b.

Mozer, F. S.: Electric Field Evidence on the Viscous Interaction at the Magnetopause, Geophys. Res. Lett., 11, 135-138, 1984.

Nykyri, K. and Otto, A.: Plasma transport at the magnetopause boundary due to reconnection in Kelvin-Helmholtz vortices, Geophys. Res. Lett., 28, 3565-3568, 2001.

Potemra, T. A., Zanetti, L. J., Bythrow, P. F., Lui, A. T. Y., and Iijima, T.: $B_{\mathrm{y}}$-Dependent Convection Patterns During Northward IMF, J. Geophys. Res., 89, 9753-9760, 1984.

Rasmussen, C. E., Schunk, R. W., and Wickwar, V. B.: A Photochemical Equilibrium Model for Ionospheric Conductivity, J. Geophys. Res., 93, 9831-9840, 1988.

Ratcliffe, J. A.: An introduction to the ionosphere and magnetosphere, Cambridge University Press, 1972.

Rees, M. H., Lummerzheim, D., Roble, R. G., Winningham, J. D., Craven, J. D., and Frank, L. A.: Auroral Energy Deposition Rate, Characteristic Electron Energy, and Ionospheric Parameters Derived From Dynamics Explorer 1 Images, J. Geophys. Res., 93, 12 841-12 860, 1988.

Reiff, P. H.: Models of Auroral-Zone Conductances, In Magnetosphere Currents (T. A. Potemra, Ed.), Geophysical Monograph 28, 180-191, American Geophysical Union, Washington, 1983.

Reiff, P. H. and Burch, J. L.: IMF $B_{y}$-Dependent Plasma Flow and Birkeland Currents in the Dayside Magnetosphere, 2. A Global Model for Northward and Southward IMF, J. Geophys. Res., 90, 1595-1609, 1985.

Reiff, P. H., Spiro, R. W., and Hill, T. W.: Dependence of Polar Cap Potential Drop on Interplanetary Parameters, J. Geophys. Res., 86, 7639-7648, 1981.

Rostoker, G., Baker, D. N., Lemaire, J., and Vasyliunas, V.: Dialog on the Relative Roles of Reconnection and the "Viscous" Interaction in Providing Solar-Wind Energy to the Magnetosphere, In Magnetotail Physics, (A. T. Y. Lui, (Ed.), 257-268, Johns Hopkins Univ. Press, Baltimore, 1987.

Russell, C. T.: The configuration of the magnetosphere, In Problems in Magnetospheric Physics, E. R. Dyer (Ed.), 1-16, National Academy of Science, Washington, 1972.

Slinker, S. P., Fedder, J. A., Emery, B. A., Baker, K. B., Lummerzheim, D., Lyon, J. G., and Rich, F. J.: Comparison of global MHD simulations with AMIE simulations for the events of 1920 May, 1996, J. Geophys. Res., 104, 28 379-28 395, 1999.

Smith, C. W., L'Heureux, J., Ness, N. F., Acuña, M. H., Burlaga, L. F., and Scheifele, J.: The ACE Magnetic Fields Experiment, Space Sci. Rev., 86, 613-632, 1998.

Sugiura, M.: A Fundamental Magnetopshere-Ionosphere Coupling Mode Involving Field-Aligned Currents as Deduced From DE-2 Observations, Geophys. Res. Lett., 1, 877-880, 1984.

Thayer, J. P.: Radar Measurements of the Electromagnetic Energy Rates Associated with the Dynamic Ionospheric Load/Generator, Geophys. Res. Lett., 25, 469-472, 1998a.

Thayer, J. P.: Height-resolved Joule heating rates in the highlatitude E region and the influence of neutral winds, J. Geophys. Res., 103, 471-487, 1998b. 
Thayer, J. P.: High-latitude currents and their energy exchange with the ionosphere-thermosphere system, J. Geophys. Res., 105, 23 015-23 024, 2000.

Thayer, J. P., Vickrey, J. F., Heelis, R. A., and Gary, J. B.: Interpretation and Modeling of the High-Latitude Electromagnetic Energy Flux, J. Geophys. Res., 100, 19715-19728, 1995.

Vaivads, A., André, M., Buchert, S., Eriksson, A. I., Olsson, A., Wahlund, J.-E., Janhunen, P., Marklund, G., Kistler, L. M., Mouikis, C., Winningham, D., Fazakerley, A., and Newell, P.: What High Altitude Observations Tell us About the Auroral Acceleration: A CLUSTER/DMSP Conjunction, Geophys. Res. Lett., 30, 1106-1109, 2003.

Waters, C. L., Anderson, B. J., and Liou, K.: Estimation of global field aligned currents using the Iridium System magnetometer data, Geophys. Res. Lett., 28, 2165-2168, 2001.

Waters, C. L., Anderson, B. J., Greenwald, R. A., Barnes, R. J., and Ruohoniemi, J. M.: High Latitude Poynting Flux From Combined Iridium and SuperDARN Data, Ann. Geophys., 22, 28612875, 2004.

SRef-ID: 1432-0576/ag/2004-22-2861.
Wygant, J. R., Torbert, R. B., and Mozer, F. S.: Comparison of S33 Polar Cap Potential Drops With the Interplanetary Magnetic Field and Models of Magnetopause Reconnection, J. Geophys. Res., 88, 5727-5735, 1983.

Zanetti, L. J., Potemra, T. A., Iijima, T., Baumjohann, W., and Bythrow, P. F.: Ionospheric and Birkeland Current Distributions for Northward Interplanetary Magnetic Field: Inferred Polar Convection, J. Geophys. Res., 89, 7453-7458, 1984.

Zanetti, L. J., Potemra, T. A., Iijima, T., Erlandson, R. E., Bythrow, P. F., Anderson, B. J., Murphree, J. S., and Marklund, G. T.: Polar Region Birkeland Current, Convection, and Aurora for Northward Interplanetary Magnetic Field, J. Geophys. Res., 95, 5825-5833, 1990. 